\title{
Nanoporous Iron Oxide/Carbon Composites through In-Situ Deposition of Prussian Blue Nanoparticles on Graphene Oxide Nanosheets and Subsequent Thermal Treatment for Supercapacitor Applications
}

\author{
Alowasheeir Azhar ${ }^{1,+}$, Yusuke Yamauchi ${ }^{2,3,4, *,+}$, Abeer Enaiet Allah ${ }^{1,5,+}$, Zeid A. Alothman $6, * \mathbb{C}$, \\ Ahmad Yacine Badjah ${ }^{6}$, Mu. Naushad ${ }^{6}\left(\mathbb{D}\right.$, Mohamed Habila ${ }^{6}$, Saikh Wabaidur ${ }^{6}$, Jie Wang ${ }^{1, *}$ \\ and Mohamed Barakat Zakaria $1,3,7, *$ (D) \\ 1 International Center for Materials Nanoarchitectonics (WPI-MANA), \\ National Institute for Materials Science (NIMS), 1-1 Namiki, Tsukuba, Ibaraki 305-0044, Japan; \\ horiatalbher@hotmail.com (A.A.); abeer.abdelaal@science.bsu.edu.eg (A.E.A.) \\ 2 Key Laboratory of Eco-Chemical Engineering, College of Chemistry and Molecular Engineering, \\ Qingdao University of Science and Technology, Qingdao 266042, China \\ 3 School of Chemical Engineering and Australian Institute for Bioengineering and Nanotechnology (AIBN), \\ The University of Queensland, Brisbane, QLD 4072, Australia \\ 4 Department of Plant \& Environmental New Resources, Kyung Hee University, 1732 Deogyeong-daero, \\ Giheung-gu, Yongin-si, Gyeonggi-do 446-701, Korea \\ 5 Chemistry Department, Faculty of Science, Beni-Suef University, Beni-Suef 62511, Egypt \\ 6 Advanced Material Research Chair, Chemistry Department, College of Science, King Saud University, \\ P.O. Box 2455, Riyadh 11451, Saudi Arabia; ybadjah@ksu.edu.sa (A.Y.B.); mnaushad@ksu.edu.sa (M.N.); \\ mhabila@ksu.edu.sa (M.H.); tarabai22@yahoo.com.sg (S.W.) \\ 7 Department of Chemistry, Faculty of Science, Tanta University, Tanta, Gharbeya 31527, Egypt \\ * Correspondence: y.yamauchi@uq.edu.au (Y.Y.); zaothman@ksu.edu.sa (Z.A.A.); \\ WANG.Jie@nims.go.jp (J.W.); mohamed.barakat@nims.go.jp (M.B.Z.) \\ $\dagger$ These authors equally contributed to this work.
}

Received: 20 March 2019; Accepted: 8 May 2019; Published: 21 May 2019

\begin{abstract}
This work reports the successful preparation of nanoporous iron oxide/carbon composites through the in-situ growth of Prussian blue (PB) nanoparticles on the surface of graphene oxide (GO) nanosheets. The applied thermal treatment allows the conversion of PB nanoparticles into iron oxide $\left(\mathrm{Fe}_{2} \mathrm{O}_{3}\right)$ nanoparticles. The resulting iron oxide/carbon composite exhibits higher specific capacitance at all scan rates than pure $\mathrm{GO}$ and $\mathrm{Fe}_{2} \mathrm{O}_{3}$ electrodes due to the synergistic contribution of electric double-layer capacitance from $\mathrm{GO}$ and pseudocapacitance from $\mathrm{Fe}_{2} \mathrm{O}_{3}$. Notably, even at a high current density of $20 \mathrm{~A} \mathrm{~g}^{-1}$, the iron oxide/carbon composite still shows a high capacitance retention of $51 \%$, indicating that the hybrid structure provides a highly accessible path for diffusion of electrolyte ions.
\end{abstract}

Keywords: nanoporous materials; iron oxide; carbon composites; Prussian blue; supercapacitors

\section{Introduction}

Hybrid materials have been extensively studied for energy storage applications due to the possibility of combining the advantages of two (or more) individual constituents to meet the demand for recent technological applications [1,2]. In recent years, coordination polymers (CPs), especially Prussian Blue (PB) and its analogues (PBAs), have been widely used for energy storage applications because of their unique features, such as their open frameworks, high stability, and redox activity. 
However, the poor conductivity of these materials presents a major obstacle for practical use. Hybrid materials composed of PB and rGO nanosheets have been investigated to some extent recently [2-4].

In general, carbon-based materials [5], such as graphene oxide (GO), reduced graphene oxide (rGO), and carbon nanotubes, or conducting polymers, such as polypyrrole (PPy) and polyaniline (PANI), are typically used to improve the conductivity of PB-based materials [6-10]. The active functional groups on the basal planes and edges of GO nanosheets allow for the adsorption of cations which act as nucleation centers for the growth of $\mathrm{PB}$ nanoparticles, thus making them attractive to be hybridized with PB. In addition, the developed reduced GO (rGO) nanosheets during composites synthesis possess high electrical conductivity and improved structural stability. Our group has reported the in-situ growth of various cyano-bridged CPs on the surface of GO by different methods [11].

PB and PBAs have been utilized as precursors for the preparation of metal oxides with good electrochemical performance for energy storage applications by means of thermal decomposition [12]. In particular, iron oxides $\left(\mathrm{Fe}_{x} \mathrm{O}_{y}\right)$ have shown some promise as an electrode material for supercapacitors owing to their high theoretical specific capacitance, cost-effectiveness, low toxicity, excellent safety, and wide abundance $[13,14]$. In this work, we demonstrate the facile preparation of a $\mathrm{PB} / \mathrm{GO}$ composite through the in-situ deposition of PB nanoparticles on GO nanosheets and the subsequent thermal treatment to convert the $\mathrm{PB} / \mathrm{GO}$ composite to nanoporous $\mathrm{Fe}_{2} \mathrm{O}_{3} /$ carbon composite (Scheme 1). The resulting $\mathrm{Fe}_{2} \mathrm{O}_{3} /$ carbon composite shows high specific capacitance, excellent rate capability, and good cycling stability for supercapacitors.

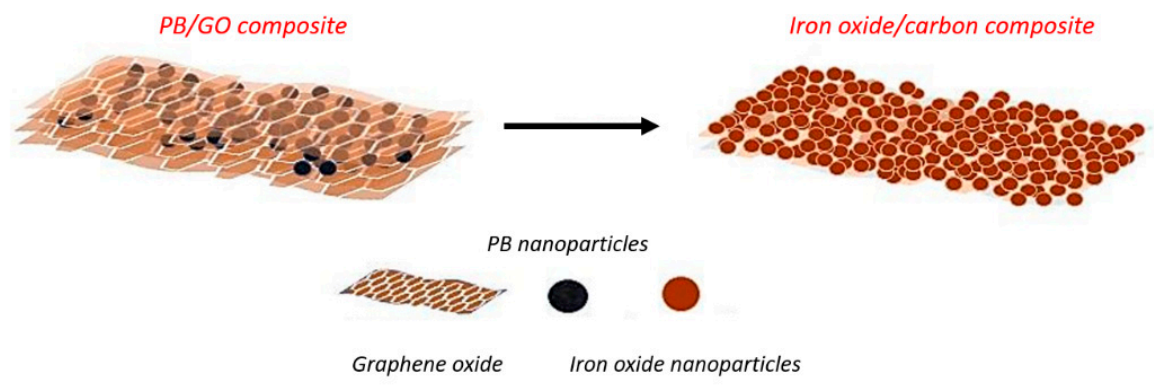

Scheme 1. Schematic illustration for the preparation of the Prussian blue/graphene oxide (PB/GO) composite and the subsequent thermal conversion to nanoporous iron oxide/carbon ( $\mathrm{Fe}_{2} \mathrm{O}_{3} /$ carbon) composite.

\section{Experimental Section}

\subsection{Chemicals}

Sodium ferrocyanide (II) decahydrate $\left(\mathrm{Na}_{4}\left[\mathrm{Fe}(\mathrm{CN})_{6}\right] \cdot 10 \mathrm{H}_{2} \mathrm{O}\right)$ was purchased from Sigma-Aldrich, Co., St. Louis, MO, USA. The sulfuric acid solution was purchased from Nacalai tesque, Inc., Kyoto, Japan. Potassium hydroxide, sodium nitrate and iron (III) chloride hexahydrate $\left(\mathrm{FeCl}_{3} \cdot 6 \mathrm{H}_{2} \mathrm{O}\right)$ were purchased from FUJIFILM Wako Pure Chemicals, Osaka, Japan. Nanographite platelets (N008-100-N) of $100 \mathrm{~nm}$ thickness were used as a raw material to prepare graphene oxide (GO) sheets (Angstron materials, Dayton, $\mathrm{OH}, \mathrm{USA})$. Potassium permanganate $\left(\mathrm{KMnO}_{4}\right)$ and hydrogen peroxide $\left(\mathrm{H}_{2} \mathrm{O}_{2}\right)$ were purchased from Kanto Chemicals Co., Inc., Tokyo, Japan. All chemical reagents were used without further purification.

\subsection{Synthesis of GO Nanosheets}

GO nanosheets were prepared according to the modified Hummer's method [1]. In a typical process, graphite powder (N008-100-N, carbon source) and sodium nitrate were mixed together and then a concentrated sulphuric acid solution $(7.67 \mathrm{~mL})$ was added into this suspension under constant stirring for $1 \mathrm{~h}$. Subsequently, $\mathrm{KMnO}_{4}(1.0 \mathrm{~g})$ was added into the resulting mixture solution while maintaining the temperature at lower than $20^{\circ} \mathrm{C}$. The reaction mixture was then stirred at $35^{\circ} \mathrm{C}$ for $2 \mathrm{~h}$ 
followed by the addition of pure water $(83 \mathrm{~mL})$ under vigorous stirring. To ensure the completion of the reaction, the obtained suspension was further treated with an aqueous $\mathrm{H}_{2} \mathrm{O}_{2}$ solution $(30 \% w / w$, $1.67 \mathrm{~mL}$ ). The resulting solution was washed several times using the diluted $\mathrm{HCl}$ solution and distilled water. Finally, it was sonicated in pure water for several hours for exfoliation. The GO powder was then collected by centrifugation for $30 \mathrm{~min}$ at 14,500 rpm. The precipitate was washed again with distilled water for several times and dried naturally in air at room temperature and then, in vacuum at $60{ }^{\circ} \mathrm{C}$ overnight. The resulting GO powder was used to prepare the aqueous GO solution $\left(2 \mathrm{mg} \mathrm{mL}^{-1}\right)$ in the next step.

\subsection{In-Situ Growth of PB Nanoparticles on the Surface of GO Nanosheets (PB/GO Composite) and the Subsequent Thermal Conversion to Nanoporous Iron Oxide/Carbon Composite}

In a typical procedure, $40 \mathrm{~mL}$ of $0.299 \mathrm{mM} \mathrm{FeCl}_{3} \cdot 6 \mathrm{H}_{2} \mathrm{O}$ solution was added dropwise to the GO solution $\left(20 \mathrm{~mL}, 2 \mathrm{mg} \mathrm{mL}^{-1}\right)$ followed by stirring for $30 \mathrm{~min}$. The obtained mixture was gently mixed with a $0.358 \mathrm{mM} \mathrm{Na}_{4}\left[\mathrm{Fe}(\mathrm{CN})_{6}\right] \cdot 10 \mathrm{H}_{2} \mathrm{O}$ solution $(40 \mathrm{~mL})$ by stirring for $30 \mathrm{~min}$. The resulting suspension was aged for two days until the reaction was completed. The precipitate of the PB/GO composite was isolated by centrifugation (for $30 \mathrm{~min}$ at 14,500 rpm) and washed with water and ethanol several times. The precipitate was left to dry at room temperature for the next step. The nanoporous $\mathrm{Fe}_{2} \mathrm{O}_{3} / \mathrm{carbon}$ composite was obtained by heating the as-synthesized $\mathrm{PB} / \mathrm{GO}$ powder at $350{ }^{\circ} \mathrm{C}$ for $1 \mathrm{~h}$ in air with a fixed heating rate of $5{ }^{\circ} \mathrm{C} \mathrm{min}^{-1}$. The $\mathrm{PB}$ nanoparticles and $\mathrm{GO}$ nanosheets were also heated under the same conditions to compare their electrochemical performance with the $\mathrm{Fe}_{2} \mathrm{O}_{3} /$ carbon composite. For the synthesis of PB nanoparticles, a $40 \mathrm{~mL}$ aqueous solution of $0.299 \mathrm{mM} \mathrm{FeCl} \cdot 6 \mathrm{H}_{2} \mathrm{O}$ was mixed with another $40 \mathrm{~mL}$ aqueous solution of $0.358 \mathrm{mM} \mathrm{Na} 4\left[\mathrm{Fe}(\mathrm{CN})_{6}\right] \cdot 10 \mathrm{H}_{2} \mathrm{O}$ and stirred for $1 \mathrm{~h}$ before aging overnight to ensure a complete reaction. The PB nanoparticles were collected by centrifugation (for $30 \mathrm{~min}$ at 14,500 rpm) and washed with water and ethanol several times. The precipitate dried naturally at room temperature (Figure S1).

\subsection{Characterization}

Wide-angle powder X-ray diffraction (PXRD) analysis was performed with Rigaku (Tokyo, Japan) RINT 2500X diffractometer using a monochromated $\mathrm{Cu} \mathrm{K} \alpha(1.5406 \AA$, $40 \mathrm{kV}, 40 \mathrm{~mA})$ radiation. Nitrogen adsorption-desorption isotherms were obtained with a Quantachrome (Boynton Beach, FL, USA) Autosorb automated gas sorption system at $77 \mathrm{~K}$. Before the measurement, all samples were dehydrated completely by heating at $250{ }^{\circ} \mathrm{C}$ overnight under vacuum. Morphological observations of the samples were performed using a scanning electron microscope (SEM, Hitachi SU8000, Tokyo, Japan) and transmission electron microscope (TEM, JEOL JEM2100, Tokyo, Japan). Fourier-transform infrared (FTIR) spectroscopy was performed using a Thermoscientific Nicolet 4700 (Waltham, MA, USA).

\subsection{Electrochemical Measurements}

All electrochemical measurements were carried out on an electrochemical analyzer $(\mathrm{CH}$ Instruments Model 600E, Austin, TX, USA). The electrochemical measurements of the prepared electrodes were investigated by the cyclic voltammetry (CV) and galvanostatic charge-discharge (GCD) using a three-electrode system with platinum wire as the counter electrode, $\mathrm{Ag} / \mathrm{AgCl}$ electrode as the reference electrode, and $3 \mathrm{M} \mathrm{KOH}$ solution as the electrolyte. The working electrode was prepared by coating a slurry containing the active material (1 mg, $85 \mathrm{wt} . \%)$, carbon black (10 wt.\%), polyvinylidene fluoride binder (PVDF) (5 wt.\%), and N-methyl-2-pyrrolidone on a graphite paper (thickness: $1 \mathrm{~mm}$ ) with an area of $1 \times 1 \mathrm{~cm}^{2}$ as the current collector and dried at $60^{\circ} \mathrm{C}$. In our experiments, we used the flexible graphite paper as the current collector because of its excellent electrical conductivity as well as its negligible capacitive performance (usually below $1 \mathrm{~F} \mathrm{~g}^{-1}$ ), which would contribute little to the total 
capacitance of the prepared working electrode. The specific gravimetric capacitance of the electrode was calculated from the CV curves by using the following Equation (1):

$$
C_{g}=\frac{1}{m s(V f-V i)} \int_{V i}^{V f} I(V) d v
$$

where $C_{g}$ is the gravimetric capacitance $\left(\mathrm{F} \mathrm{g} \mathrm{g}^{-1}\right), s$ is the potential scan rate $\left(\mathrm{V} \mathrm{s}^{-1}\right), V$ is the potential window $(\mathrm{V}), I$ is current $(\mathrm{A})$, and $m$ is the mass of active material $(\mathrm{g})$.

Based on galvanostatic charge-discharge (GCD) curves, the gravimetric specific capacitance $\left(C_{g}\right.$, $\mathrm{F}^{-1}$ ) was calculated using the Equation (2):

$$
C_{g}=\frac{I \cdot t}{m \cdot \Delta V}
$$

where $I$ is the discharge current $(\mathrm{A}), t$ is the discharge time $(\mathrm{s}), m$ is the mass of active material $(\mathrm{g})$, and $\Delta V$ is the potential change during the discharge process $(\mathrm{V})$.

\section{Results and Discussion}

The functional groups, including hydroxyl, carboxyl and carbonyl on the GO nanosheets enables the adsorption of $\mathrm{Fe}^{3+}$ ions at the GO surface in the first step. Then, the adsorbed $\mathrm{Fe}^{3+}$ ions interact with the ferrocyanide ligand to initiate the growth of PB nanoparticles on the GO surface. The change in the surface charge of the GO nanosheet suspension before and after the addition of $\mathrm{Fe}^{3+}$ ions was studied by measuring the zeta potentials. The zeta potential measurements reveal that the surface charge of the GO nanosheets was changed from negative to positive due to the adsorbed cations $\left(\mathrm{Fe}^{3+}\right.$ ions) $[15,16]$. During the growth of $\mathrm{PB}$, the color of the suspension gradually changed to blue due to the formation of PB nanoparticles (Figure 1a). The morphology of the PB/GO composite was investigated by both SEM and TEM (Figure $1 b, c)$. The high-magnification SEM and TEM images of the PB/GO composite clearly show the wrinkly structure of GO-wrapped PB nanoparticles.
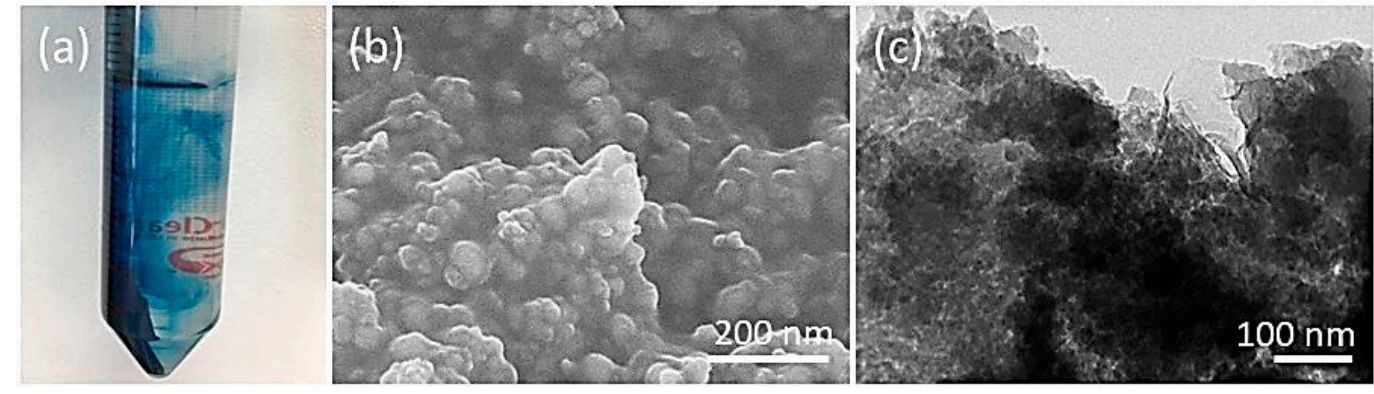

Figure 1. (a) A digital photograph of the PB/GO precipitate collected by centrifugation; (b) SEM and (c) TEM images of the as-synthesized PB/GO composite.

The crystal structure and phase purity of the PB/GO composite were checked by XRD (Figure 2a). The XRD pattern of the GO nanosheets shows two sharp diffraction peaks at $2 \theta=11^{\circ}$ and $26^{\circ}$ indexed to (001) and (002) planes of GO, respectively, indicating the highly ordered nature of the graphitic layers $[17,18]$. The XRD pattern of the PB nanoparticles (Figure 2a) is assignable to a typical face-centered cubic PB crystal structure (JCPDS No. 73-0687) [19]. The diffraction peaks of PB still remain in the $\mathrm{PB} / \mathrm{GO}$ composite even after the hybridization with $\mathrm{GO}$, however the characteristic peaks of the GO nanosheets are not detected because the anchored PB nanoparticles cover the surface of GO nanosheets, thus preventing their restacking. 

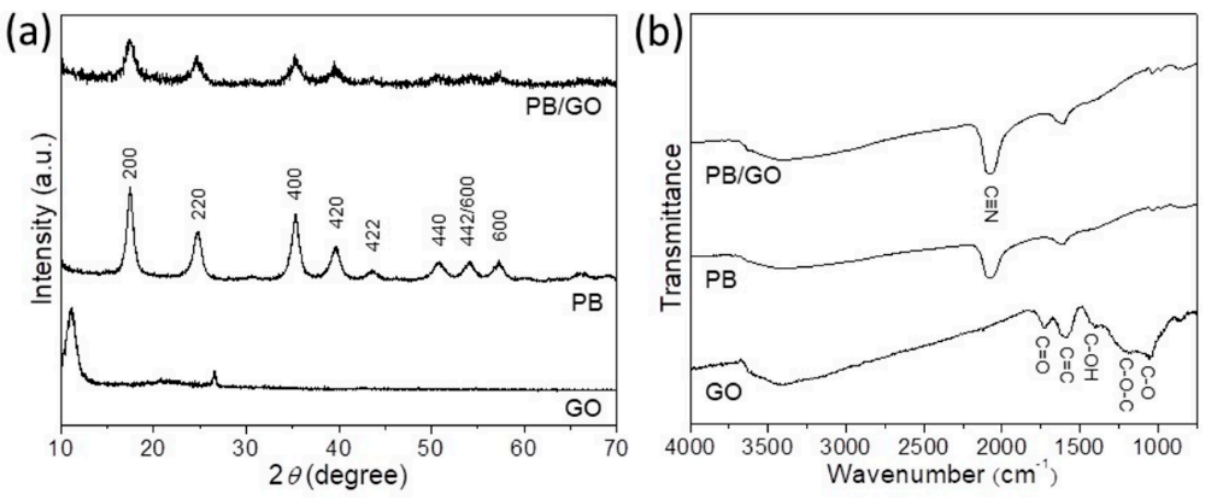

Figure 2. (a) Wide-angle XRD diffraction patterns and (b) FT-IR spectra of graphene oxide (GO) nanosheets, Prussian blue (PB) nanoparticles, and PB/GO composite.

The chemical compositions of the PB nanoparticles, GO nanosheets, and PB/GO composite were investigated by the FTIR spectroscopy (Figure 2b). The peaks observed in the IR spectrum of GO nanosheets can be assigned to the oxygen-containing functional groups on the GO surface [20-22]. After the hybridization with PB nanoparticles, several bands belonging to GO disappear and/or the peak intensities are largely reduced, due to the successful reduction of GO to reduced graphene oxide (rGO). The peak at $2080 \mathrm{~cm}^{-1}$ is attributed to $-\mathrm{C} \equiv \mathrm{N}$ - units, thus confirming the existence of $\mathrm{PB}$ nanoparticles [23,24]. The peak at $1600 \mathrm{~cm}^{-1}$ on the $\mathrm{PB} / \mathrm{GO}$ composite is difficult to identify as it overlaps with the $\mathrm{C}=\mathrm{C}$ group of $\mathrm{GO}$ nanosheets and the $-\mathrm{OH}$ group of $\mathrm{PB}$ nanoparticles.

As shown in Scheme 1, the thermal conversion of the $\mathrm{PB} / \mathrm{GO}$ composite at $350{ }^{\circ} \mathrm{C}$ in air results in the formation of the iron oxide $\left(\mathrm{Fe}_{2} \mathrm{O}_{3}\right)$ /carbon composite [25]. The crystal structure of the $\mathrm{PB} / \mathrm{GO}$ composite after the heat treatment was examined by the wide-angle XRD (Figure 3a). The original peaks of $\mathrm{PB}$ vanished entirely. The new and broad peaks at $2 \theta=35^{\circ}$ and $63^{\circ}$ can be assigned to impurity-free $\gamma-\mathrm{Fe}_{2} \mathrm{O}_{3}$ [26], thereby confirming the successful conversion of PB to iron oxide. The diffraction peaks of GO nanosheets are not observed in the prepared $\mathrm{Fe}_{2} \mathrm{O}_{3}$ /carbon composite. The morphology of this composite was investigated using both SEM and TEM (Figure 3b-d). As revealed by the SEM image, the size of the resulting iron oxide nanoparticles is nearly similar as that of the starting PB nanoparticles (Figure 1b). The high-resolution SEM image shows that the iron oxide nanoparticles are aggregated to each other. To further investigate the morphology of this composite, TEM analysis was carried out. Figure $3 \mathrm{c}$ confirms the presence of nanoparticle aggregates and wrinkled GO nanosheets. The high-resolution TEM (HRTEM) image shows several lattice fringes belonging to iron oxide (Figure 3d). The EDX elemental mapping shows the uniform distribution of carbon $(\mathrm{C})$, oxygen $(\mathrm{O})$, and iron (Fe) atoms (Figure 4). The Fe:C ratio of the resulting iron oxide/carbon composite is around 62:38 (wt.\%). The presence of Fe and C was also confirmed by FTIR spectroscopy, as shown in Figure S2. 

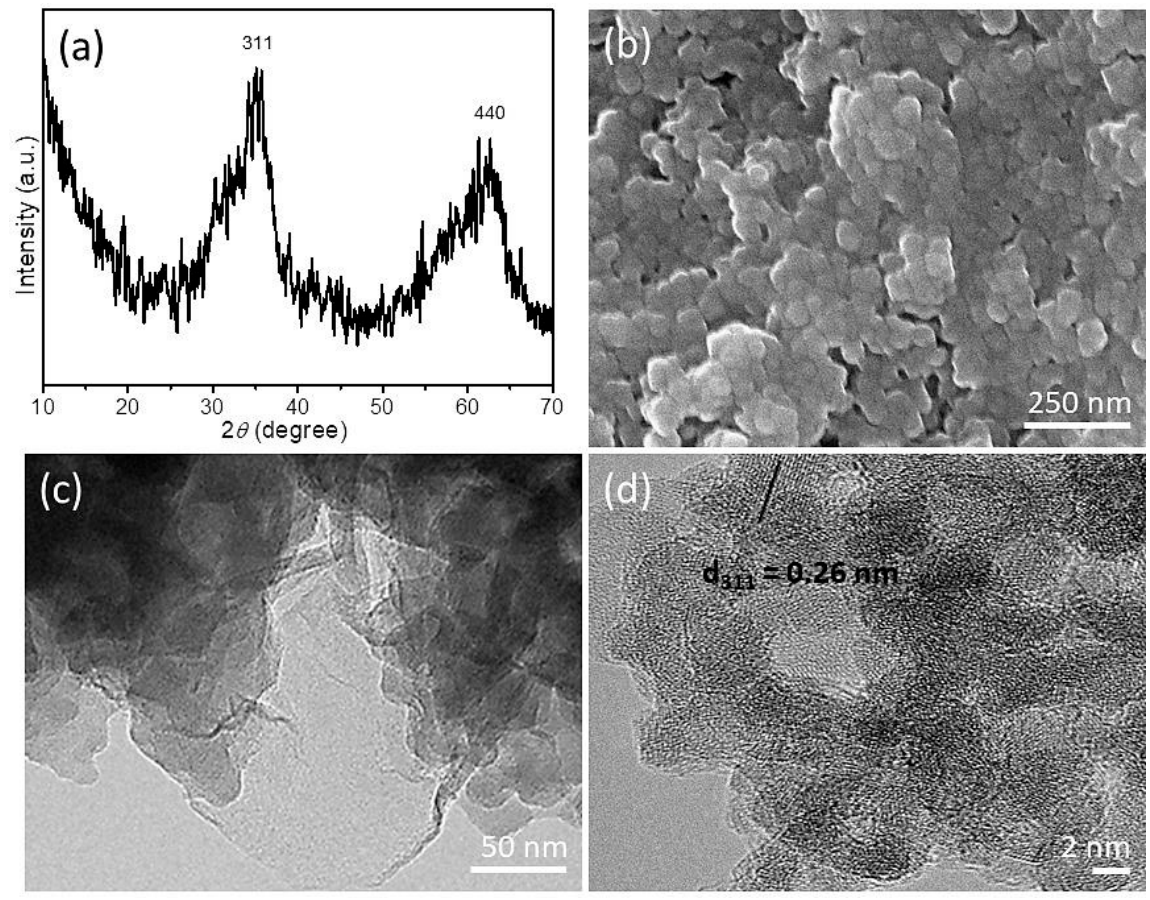

Figure 3. (a) Wide-angle XRD pattern, and (b) SEM, (c) TEM and (d) high-resolution TEM (HRTEM) images of the iron oxide/carbon composite obtained by the thermal treatment of the PB/GO composite.
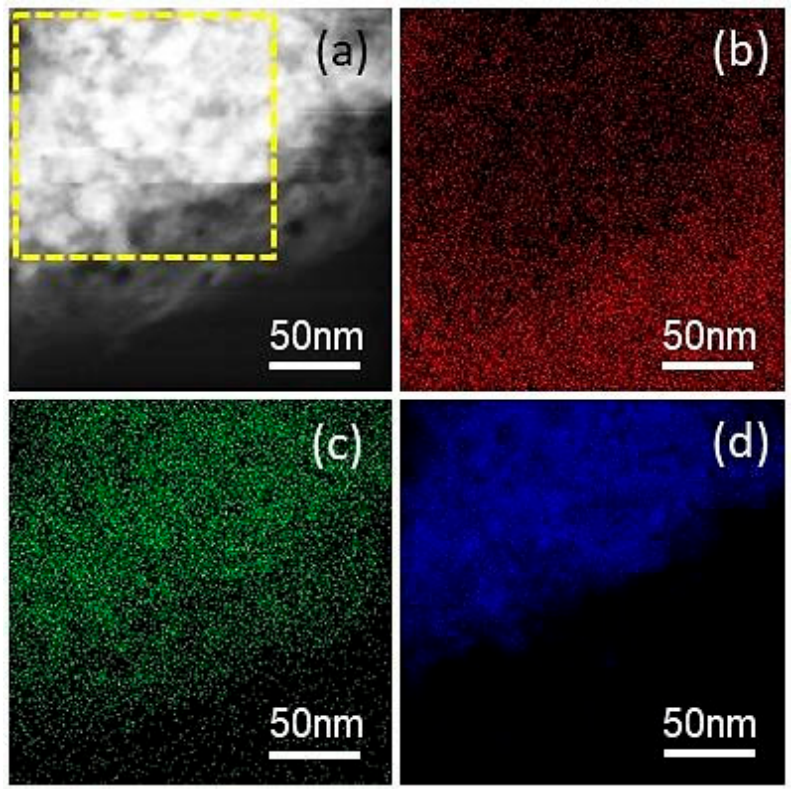

Figure 4. (a) HAADF-STEM image of the iron oxide/carbon composite and the corresponding elemental mapping for (b) carbon, (c) oxygen, and (d) iron contents. The weight ratio of carbon and iron was measured from the selected area highlighted in the yellow box.

Figure 5 shows the $\mathrm{N}_{2}$ adsorption-desorption isotherms of $\mathrm{PB}$ nanoparticles and $\mathrm{PB} / \mathrm{GO}$ composite before and after thermal treatment. The textural characteristics are summarized in Table 1. The Brunauer-Emmett-Teller (BET) specific surface area of the PB/GO composite $\left(152.6 \mathrm{~m}^{2} \mathrm{~g}^{-1}\right)$ is remarkably improved compared to pure GO $\left(34.9 \mathrm{~m}^{2} \mathrm{~g}^{-1}\right)$ [1] and pure $\mathrm{PB}\left(36.1 \mathrm{~m}^{2} \mathrm{~g}^{-1}\right)$. The adsorption isotherms are gradually increased with the increase of relative pressure, indicating that various pores with different pore sizes are randomly distributed over the entire area. The higher surface area of the $\mathrm{PB} / \mathrm{GO}$ composite can be attributed to the good dispersion of the $\mathrm{PB}$ nanoparticles on the GO 
surface, thus enabling them to act as spacers to prevent the GO layers from aggregation or restacking. Following the heat treatment, the surface area of the iron oxide/carbon composite is mostly similar to that of the precursor PB/GO composite.

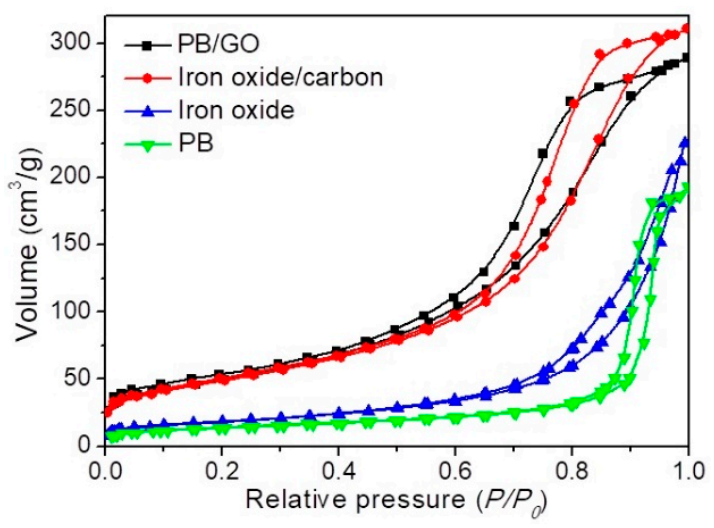

Figure 5. Nitrogen gas adsorption-desorption isotherms of PB nanoparticles, iron oxide nanoparticles, $\mathrm{PB} / \mathrm{GO}$ composite, and iron oxide/carbon composite.

Table 1. Textural characteristics of the obtained samples.

\begin{tabular}{ccc}
\hline Sample & Surface Area $/ \mathbf{m}^{\mathbf{2}} \mathbf{g}^{\mathbf{- 1}}$ & Pore Volume $/ \mathbf{c m}^{\mathbf{3}} \mathbf{g}^{\mathbf{1}}$ \\
\hline PB & 36.1 & 0.291 \\
Iron oxide & 60.3 & 0.343 \\
PB/GO & 152.6 & 0.453 \\
Iron oxide/carbon & 145.5 & 0.487 \\
\hline
\end{tabular}

It is well-reported that graphene/metal oxide composites exhibit good electrochemical performance for supercapacitor applications $[27,28]$. The electrochemical properties of pure GO, pure iron oxide, and iron oxide/carbon composite electrodes for supercapacitor applications were investigated by using a three-electrode system in a $3 \mathrm{M} \mathrm{KOH}$ electrolyte. The cyclic voltammetry (CV) curves of the pure GO electrode at different scan rates (Figure 6a) reveal rectangle-like curves, indicating its electrical double layer capacitive properties, while in the case of iron oxide and iron oxide/carbon electrodes (Figure 6b,c) the CV curves exhibit a pair of redox peaks due to pseudocapacitive behavior. The existence of an oxidation peak at ca. $0.3 \mathrm{~V}$ and a reduction peak at ca. $-0.7 \mathrm{~V}$ vs. $\mathrm{Ag} / \mathrm{AgCl},[29]$ for the iron oxide/carbon composite electrode implies reversible redox reactions of iron ion [30].

Based on CV measurements, the specific capacitance values of GO, iron oxide, and iron oxide/carbon electrodes at a scan rate of $2 \mathrm{mV} \mathrm{s}^{-1}$ are 220.0, 264.0, and $551.5 \mathrm{~F} \mathrm{~g}^{-1}$, respectively. More importantly, the iron oxide/carbon composite electrode exhibits higher specific capacitance values than both GO and iron oxide electrodes at all scan rates owing to the synergistic combination of EDLC from GO and pseudocapacitance from $\mathrm{Fe}_{2} \mathrm{O}_{3}$ (Table 2). The capacitance retention value of the iron oxide/carbon composite electrode at $100 \mathrm{mV} \mathrm{s}^{-1}$ is very high (50\%), superior to those of graphene oxide (31\%) and iron oxide electrodes (44\%). 

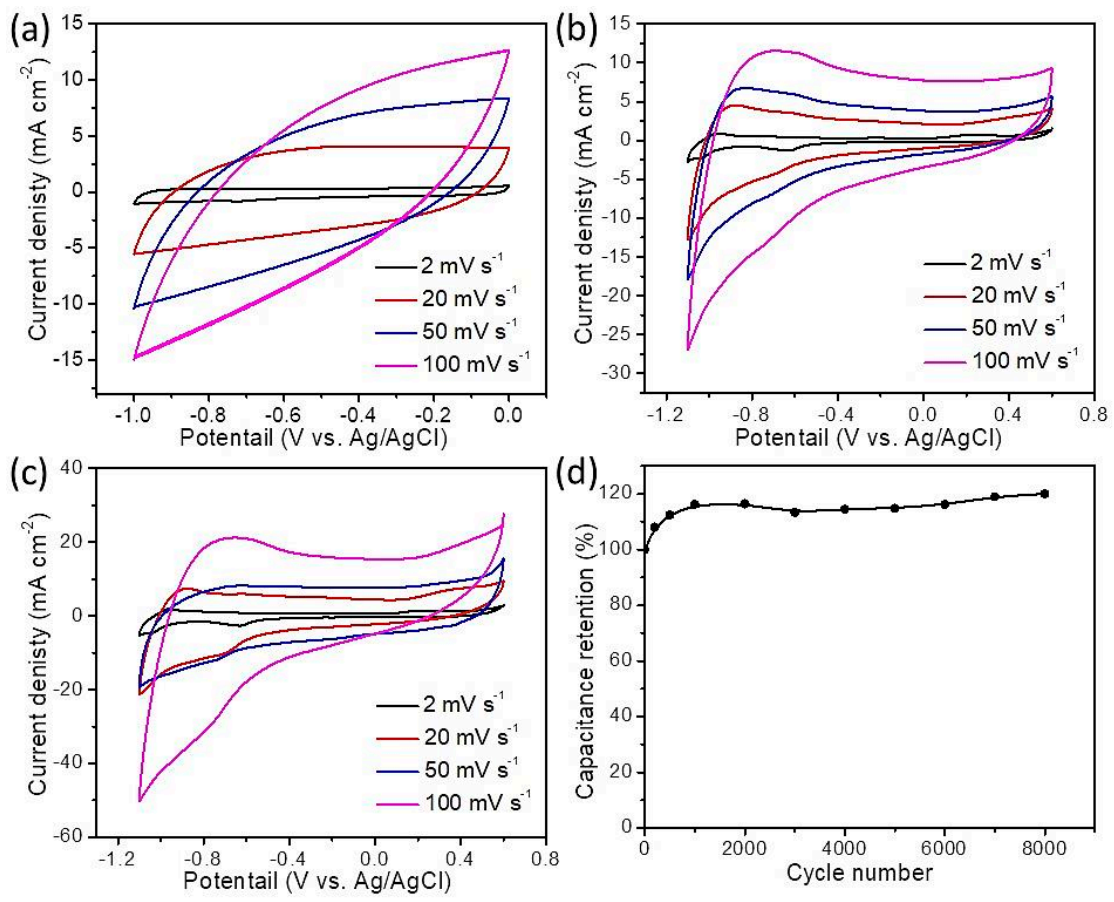

Figure 6. (a-c) CV curves at various scan rates for (a) graphene oxide; (b) iron oxide and (c) iron oxide/carbon electrodes; (d) cycling performances at $200 \mathrm{mV} \mathrm{s}^{-1}$ for the iron oxide/carbon composite electrode.

Table 2. Specific capacitance values of GO, iron oxide, and iron oxide/carbon composite at different scan rates.

\begin{tabular}{cccc}
\hline \multirow{2}{*}{ Scan Rate/mV s } & & \multicolumn{2}{c}{ Specific Capacitance/F $\mathbf{~ g}^{-\mathbf{1}}$} \\
& GO & Iron Oxide & Iron Oxide/Carbon \\
\hline 2 & 220.0 & 264.0 & 551.5 \\
20 & 205.3 & 145.5 & 450.0 \\
50 & 110.0 & 91.70 & 340.0 \\
100 & 97.00 & 82.00 & 275.0 \\
\hline
\end{tabular}

The long-term stability of the iron oxide/carbon composite electrode was investigated by the $\mathrm{CV}$ measurement at $100 \mathrm{mV} \mathrm{s}^{-1}$ (Figure 6d). Clearly, the capacitance gradually increases until the capacitance retention reaches $120 \%$ after 8000 cycles. The increase in the specific capacitance during cycling is possibly due to the activation of the hybrid electrode material as a result of the improved surface wetting of the electrode, leading to improvement in the electrolyte ion diffusion. Furthermore, the as-prepared iron oxide/carbon composite electrode shows better electrochemical performance for supercapacitor applications than previously reported iron oxide/GO electrodes in terms of specific capacitance and capacitance retention (Table S1).

Galvanostatic charge-discharge (GCD) measurements were carried out to investigate the rate performance of the graphene oxide, iron oxide, and iron oxide/carbon electrodes at current densities of $2,4,6,8,10$, and $20 \mathrm{~A} \mathrm{~g}^{-1}$ (Figure 7). The GCD curves of the iron oxide/carbon composite electrode depicts high specific capacitance in comparison with graphene oxide and iron oxide. A non-linear behavior was observed during charging (at a potential of ca. $0.3 \mathrm{~V}$ ) and discharging (at a potential of ca. $-0.7 \mathrm{~V}$ ) vs. $\mathrm{Ag} / \mathrm{AgCl}$ and these observations are in good agreement with the oxidation and reduction peaks observed in the $\mathrm{CV}$ curves. Figure $7 \mathrm{~d}$ compares the rate capabilities of graphene oxide, iron oxide, and iron oxide/carbon composite electrodes at different current densities. At a current density of $2 \mathrm{~A} \mathrm{~g}^{-1}$, the specific capacitance of the iron oxide/carbon composite electrode is $415.0 \mathrm{~F} \mathrm{~g}^{-1}$, which is much higher than those of graphene oxide $\left(210.0 \mathrm{~F} \mathrm{~g}^{-1}\right)$ and iron oxide electrodes $\left(202.5 \mathrm{~F} \mathrm{~g}^{-1}\right)$. 
Notably, with the increase of current density to $20 \mathrm{~A} \mathrm{~g}^{-1}$, the iron oxide/carbon composite electrode still exhibits a high capacitance retention of $51 \%$. On the contrary, pure graphene oxide and iron oxide electrodes display poor capacitance retention of $21 \%$ and $29 \%$, respectively, indicating that the hybrid structure was beneficial for enhancing the diffusion of electrolyte ions.
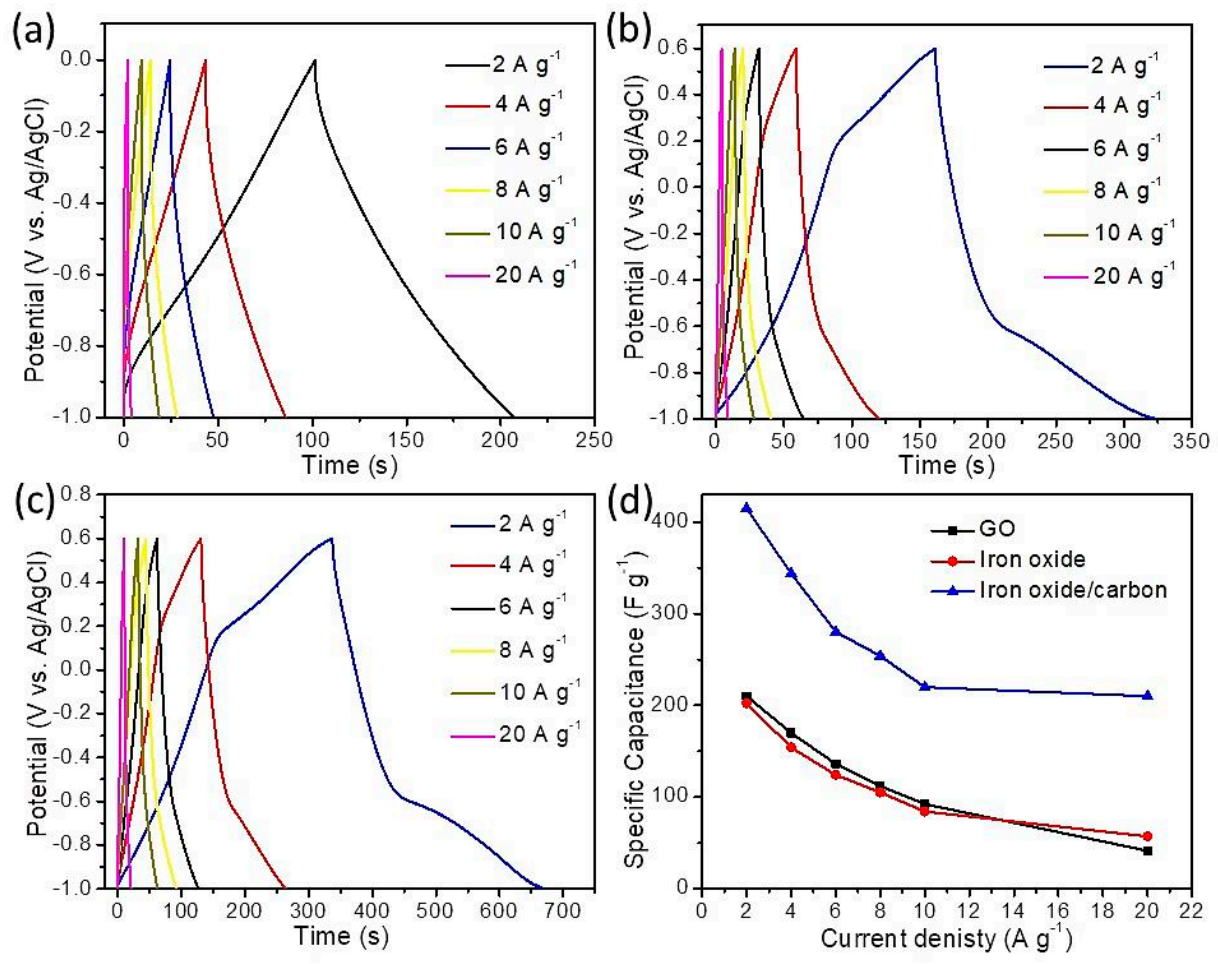

Figure 7. Galvanostatic charge-discharge (GCD) curves of (a) graphene oxide; (b) iron oxide and (c) iron oxide/carbon electrodes at different current densities; (d) specific capacitance compared to current density plots for all the samples.

\section{Conclusions}

In this study, PB nanoparticles were simultaneously deposited on the surface of GO nanosheets through the adsorption of positively charged $\mathrm{Fe}^{3+}$ ions on $\mathrm{GO}$ surface firstly followed by addition of $\mathrm{Na}_{4}\left[\mathrm{Fe}(\mathrm{CN})_{6}\right] \cdot 10 \mathrm{H}_{2} \mathrm{O}$. The oxygen-containing functional groups of the GO surface served as excellent anchoring agents for the growth of PB nanoparticles. The PB/GO composite precursor was successfully converted into the iron oxide/GO composite with a well-retained morphology by a facile thermal annealing in air at $350{ }^{\circ} \mathrm{C}$. The obtained iron oxide/carbon composite shows superior electrochemical performance as an electrode material for supercapacitors compared to both pure iron oxide and graphene oxide electrodes in terms of specific capacitance, rate capability, and capacitance retention. The results will provide a useful guidance for constructing high-performance hybrid materials for energy storage applications using PB and PBA.

Supplementary Materials: The following are available online at http://www.mdpi.com/2079-4991/9/5/776/s1, Figure S1: (a) SEM and (b) TME images of PB nanoparticles; Figure S2: FTIR spectrum of iron oxide/carbon composite; Table S1: Comparison of our sample performance with the previously reported iron oxide/carbon materials composites for supercapacitors.

Author Contributions: Conceptualization, Y.Y. and M.B.Z.; methodology, A.A. and A.E.A.; validation, J.W. and M.B.Z.; formal analysis, J.W. and M.B.Z.; investigation, Y.Y., J.W. and M.B.Z.; resources, Y.Y.; data curation, Z.A.A., M.N., M.H. and M.B.Z.; writing-original draft preparation, A.A., A.E.A., A.Y.B., M.N. and M.B.Z.; writing-review and editing, Y.Y., J.W. and M.B.Z.; supervision, Y.Y. and Z.A.A.; project administration, M.H. and S.W.; funding acquisition, Z.A.A., M.H. and S.W.

Funding: This research received no external funding. 
Acknowledgments: This project was funded by the NSTIP strategic technologies program (Project No. ADV 1285-02) in the Kingdom of Saudi Arabia.

Conflicts of Interest: The authors declare no conflict of interest.

\section{References}

1. Tanaka, S.; Salunkhe, R.R.; Kaneti, Y.V.; Malgras, V.; Alshehri, S.M.; Ahamad, T.; Zakaria, M.B.; Dou, S.X.; Yamauchi, Y.; Hossain MS, A. Prussian blue derived iron oxide nanoparticles wrapped in graphene oxide sheets for electrochemical supercapacitors. RSC Adv. 2017, 7, 33994-33999. [CrossRef]

2. Wang, Z.L.; Sun, K.; Henzie, J.; Hao, X.; Ide, Y.; Takei, T.; Bando, Y.; Yamauchi, Y. Electrochemically in situ controllable assembly of hierarchically-ordered and integrated inorganic-carbon hybrids for efficient hydrogen evolution. Mater. Horiz. 2018, 5, 1194-1203. [CrossRef]

3. Sun, D.; Wang, H.; Deng, B.; Zhang, H.; Wang, L.; Wan, Q.; Yan, X.; Qu, M. A Mn Fe based Prussian blue Analogue@Reduced graphene oxide composite as high capacity and superior rate capability anode for lithium-ion batteries. Carbon 2019, 143, 706-713. [CrossRef]

4. Cao, L.; Liu, Y.; Zhang, B.; Lu, L. In situ controllable growth of prussian blue nanocubes on reduced graphene oxide: Facile synthesis and their application as enhanced nanoelectrocatalyst for $\mathrm{H}_{2} \mathrm{O}_{2}$ reduction. ACS Appl. Mater. Interfaces 2010, 2, 2339-2346. [CrossRef] [PubMed]

5. Jiang, H.; Leeb, P.S.; Li, C. 3D carbon based nanostructures for advanced supercapacitors. Energy Environ. Sci. 2013, 6, 41-53. [CrossRef]

6. Wong, M.H.; Zhang, Z.; Yang, X.; Chen, X.; Ying, J.Y. One-pot in situ redox synthesis of hexacyanoferrate/conductive polymer hybrids as lithium-ion battery cathodes. Chem. Commun. 2015, 51, 13674-13677. [CrossRef] [PubMed]

7. Wang, X.J.; Krumeich, F.; Nesper, R. Nanocomposite of manganese ferrocyanide and graphene: A promising cathode material for rechargeable lithium ion batteries. Electrochem. Commun. 2013, 34, 246-249. [CrossRef]

8. Daneshvar, F.; Aziz, A.; Abdelkader, A.M.; Zhang, T.; Sue, H.-J.; Welland, M.E. Porous $\mathrm{SnO}_{2}-\mathrm{Cu}_{\mathrm{x}} \mathrm{O}$ nanocomposite thin film on carbon nanotubes as electrodes for high performance supercapacitors. Nanotechnology 2019, 30, 015401. [CrossRef] [PubMed]

9. Chou, S.-L.; Wang, J.-Z.; Chew, S.-Y.; Liu, H.-K.; Dou, S.-X. Electrodeposition of $\mathrm{MnO}_{2}$ nanowires on carbon nanotube paper as free-standing, flexible electrode for supercapacitors. Electrochem Commun. 2008, 10, 1724-1727. [CrossRef]

10. Chen, Z.; Augustyn, V.; Wen, J.; Zhang, Y.; Shen, M.; Dunn, B.; Lu, Y. High-Performance Supercapacitors Based on Intertwined $\mathrm{CNT} / \mathrm{V}_{2} \mathrm{O}_{5}$ Nanowire Nanocomposites. Adv. Mater. 2011, 23, 791-795. [CrossRef]

11. Zakaria, M.B.; Tan, H.; Kim, J.; Badjah, A.Y.; Naushad, M.; Habila, M.; Wabaidur, S.; Alothman, Z.A.; Yamauchi, Y.; Lin, J. Structurally controlled layered $\mathrm{Ni}_{3} \mathrm{C}$ /graphene hybrids using cyano-bridged coordination polymers. Electrochem. Commun. 2019, 100, 74-8075. [CrossRef]

12. Cai, Z.X.; Wang, Z.L.; Kim, J.; Yamauchi, Y. Hollow Functional Materials Derived from Metal-Organic Frameworks: Synthetic Strategies, Conversion Mechanisms, and Electrochemical Applications. Adv. Mater. 2019, 31, 1804903. [CrossRef]

13. Tanaka, S.; Zakaria, M.B.; Kaneti, Y.V.; Jikihara, Y.; Nakayama, T.; Zaman, M.; Bando, Y.; Hossain, M.S.A. Gold-loaded nanoporous iron oxide cubes derived from Prussian blue as carbon monoxide oxidation catalyst at room temperature. ChemistrySelect 2018, 3, 13464-13469. [CrossRef]

14. Liu, H.D.; Zhang, J.L.; Xu, D.D.; Huang, L.H.; Tan, S.Z.; Mai, W.J. Easy one-step hydrothermal synthesis of nitrogen-doped reduced graphene oxide/iron oxide hybrid as efficient supercapacitor material. J. Solid State Electrochem. 2014, 19, 135-144. [CrossRef]

15. Zakaria, M.B.; Li, C.; Ji, Q.; Jiang, B.; Tominaka, S.; Ide, Y.; Hill, J.P.; Ariga, K.; Yamauchi, Y. Self-construction from 2D to 3D: One-Pot layer-by-layer assembly of graphene oxide sheets held together by coordination polymers. Angew. Chem. Int. Ed. 2016, 55, 8426-8430. [CrossRef] [PubMed]

16. Azhar, A.; Zakaria, M.B.; Lin, J.; Chikyow, T.; Martin, D.J.; Alghamdi, Y.G.; Alshehri, A.A.; Bando, Y.; Hossain, M.S.A.; Wu, K.C.W.; et al. Graphene-wrapped nanoporous nickel-cobalt oxide flakes for electrochemical supercapacitors. ChemistrySelect 2018, 3, 8505-8510. [CrossRef] 
17. Chen, Y.L.; Hu, Z.A.; Chang, Y.Q.; Wang, H.W.; Zhang, Z.Y.; Yang, Y.Y.; Wu, H.Y. Zinc oxide/reduced graphene oxide composites and electrochemical capacitance enhanced by homogeneous incorporation of reduced graphene oxide sheets in zinc oxide matrix. J. Phys. Chem. C 2011, 115, 2563-2571. [CrossRef]

18. Huang, X.; Zhi, C.; Jiang, P.; Golberg, D.; Bando, Y.; Tanaka, T. Temperature-dependent electrical property transition of graphene oxide paper. Nanotechnology 2012, 23, 455705. [CrossRef]

19. Muthusamy, S.; Charles, J.; Renganathan, B.; Sastikumar, D. In situ growth of Prussian blue nanocubes on polypyrrole nanoparticles: Facile synthesis, characterization and their application as fiber optic gas sensor. $J$. Mater. Sci. 2018, 53, 15401-15417. [CrossRef]

20. Vermisoglou, E.C.; Devlin, E.; Giannakopoulou, T.; Romanos, G.; Boukos, N.; Psycharis, V.; Lei, C.; Lekakou, C.; Petridis, D.; Trapalis, C. Reduced graphene oxide/iron carbide nanocomposites for magnetic and supercapacitor applications. J. Alloys Compd. 2014, 590, 102-109. [CrossRef]

21. Liu, X.W.; Yao, Z.J.; Wang, Y.F.; Wei, X.W. Graphene oxide sheet-prussian blue nanocomposites: Green synthesis and their extraordinary electrochemical properties. Coll. Surf. B Biointerfaces 2010, 81, 508-512. [CrossRef] [PubMed]

22. Jang, S.C.; Haldorai, Y.; Lee, G.W.; Hwang, S.K.; Han, Y.K.; Roh, C.; Huh, Y.S. Porous three-dimensional graphene foam/Prussian blue composite for efficient removal of radioactive137Cs. Sci. Rep. 2015, 5, 17510. [CrossRef] [PubMed]

23. Wang, S.C.; Gu, M.; Pan, L.; Xu, J.; Han, L.; Yi, F.Y. The interlocked: In situ fabrication of graphene@prussian blue nanocomposite as high-performance supercapacitor. Dalton Trans. 2018, 47, 13126-13134. [CrossRef] [PubMed]

24. Yang, H.; Li, H.; Zhai, J.; Sun, L.; Zhao, Y.; Yu, H. Magnetic prussian blue/graphene oxide nanocomposites caged in calcium alginate microbeads for elimination of cesium ions from water and soil. Chem. Eng. J. 2014, 246, 10-19. [CrossRef]

25. Hu, M.; Belik, A.A.; Imura, M.; Mibu, K.; Tsujimoto, Y.; Yamauchi, Y. Synthesis of superparamagnetic nanoporous iron oxide particles with hollow interiors by using Prussian blue coordination polymers. Chem. Mater. 2012, 24, 2698-2707. [CrossRef]

26. Zakaria, M.B.; Belik, A.A.; Liu, C.; Hsieh, H.; Liao, Y.; Malgras, V.; Yamauchi, Y.; Wu, K.C. Prussian blue derived nanoporous iron oxides as anticancer drug carriers for magnetic-guided chemotherapy. Chem. Asian J. 2015, 10, 1457-1462. [CrossRef] [PubMed]

27. Ma, W.; Chen, S.; Yang, S.; Chen, W.; Weng, W.; Cheng, Y.; Zhu, M. Flexible all-solid-state asymmetric supercapacitor based on transition metal oxide nanorods/reduced graphene oxide hybrid fibers with high energy density. Carbon 2017, 113, 151-158. [CrossRef]

28. Li, M.; Pan, F.; Shi, E.; Choo, G.; Lv, Y.; Chen, Y.; Xue, J. Designed construction of a graphene and iron oxide freestanding electrode with enhanced flexible energy-storage performance. ACS Appl. Mater. Interfaces 2016, 8, 6972-6981. [CrossRef]

29. Mallick, S.; Jana, P.P.; Raj, C.R. Asymmetric supercapacitor based on chemically coupled hybrid material of $\mathrm{Fe}_{2} \mathrm{O}_{3}-\mathrm{Fe}_{3} \mathrm{O}_{4}$ heterostructure and nitrogen-doped reduced graphene oxide. ChemElectroChem 2018, 5, 2348-2356. [CrossRef]

30. Liu, M.; Sun, J. In situ growth of monodisperse $\mathrm{Fe}_{3} \mathrm{O}_{4}$ nanoparticles on graphene as flexible paper for supercapacitor. J. Mater. Chem. A 2014, 2, 12068-12074. [CrossRef]

(C) 2019 by the authors. Licensee MDPI, Basel, Switzerland. This article is an open access article distributed under the terms and conditions of the Creative Commons Attribution (CC BY) license (http://creativecommons.org/licenses/by/4.0/). 